\title{
Microarray-based identification of nerve growth-promoting genes in neurofibromatosis type I
}

\author{
YUFEI LIU ${ }^{1 *}$, DONG KANG ${ }^{2 *},{\text { CHUNYAN } \mathrm{LI}^{3}, \text { GUANG XU }^{4}, \text { YAN TAN }^{5} \text { and JINHONG WANG }}^{6}$ \\ ${ }^{1}$ Department of Pediatric Intensive Care Unit, The First Affiliated Hospital of Jilin University, Changchun, \\ Jilin 130000; ${ }^{2}$ Huiqiao Department, South Hospital of Southern Medical University, Guangdong 510630; \\ Departments of ${ }^{3}$ Pediatric Respiratory and ${ }^{4}$ Infectious Diseases, The First Affiliated Hospital of Jilin University; \\ ${ }^{5}$ Cancer Biotherapy Center, People's Hospital of Jilin Province, Changchun, Jilin 130000; ${ }^{6}$ Department of Respiratory, \\ The Third Affiliated Hospital of Southern Medical University, Guangzhou, Guangdong 510630, P.R. China
}

Received May 16, 2013; Accepted October 29, 2013

DOI: $10.3892 / \mathrm{mmr} .2013 .1785$

\begin{abstract}
As a genetic disease, neurofibromatosis type 1 (NF1) is characterized by abnormalities in multiple tissues derived from the neural crest, including neoplasms in the ends of the limbs. The exact mechanism of nerve growth in NF1 is unclear. In the present study, the gene expression profile of nerves in healthy controls and NF1 patients with macrodactylia of the fingers or toes were analyzed in order to identify possible genes associated with nerve growth. The Whole Human Genome Microarray was selected to screen for different gene expression profiles, and the result was analyzed with Feature Extraction software. The target genes were verified at the transcriptional and translational levels by reverse transcription-polymerase chain reaction and western blotting, respectively. A common set of 28 genes were identified to be changed $>5$-fold in the NF1 patients compared with the healthy controls. Among them, the metastasis-associated genes, lymphocyte function-associated antigen $1, \mathrm{C}-\mathrm{X}$-C chemokine receptor type 4 and intracellular adhesion molecule 1 , and the inflammatory cytokines, interleukin (IL) $1 \beta$, IL-6 and IL-8, were downregulated significantly. Mainly genes that changed $>10$-fold were analyzed in this study, and HOXC 8 demonstrated activity in promoting nerve growth. Through the analysis of the mRNA expression of the nerves in the NF1
\end{abstract}

Correspondence to: Dr Jinhong Wang, Department of Respiratory, The Third Affiliated Hospital of Southern Medical University, 183 East Zhongshan Road, Guangzhou, Guangdong 510630, P.R. China

E-mail: leechy_wangjh@163.com

Dr Yan Tan, Cancer Biotherapy Center, People's Hospital of Jilin Province, 1183 Gongnong Road, Changchun, Jilin 130000, P.R.China

E-Mail: stone_tany@163.com

*Contributed equally

Key words: neurofibromatosis type I, microarray analysis, gene expression patients, target molecules contributing to nerve growth during NF1 development were investigated, which aided in improving our understanding of this disease, and may provide a novel direction for nerve repair and regeneration.

\section{Introduction}

Neurofibromatosis is characterized by café-au-lait spots, iris Lisch nodules and cutaneous or subcutaneous skin tumors termedneurofibromas (1). This disease may cause tissue along the nerves to grow uncontrollably, inducing serious damage by compressing the nerves. Two types of neurofibromatosis, type 1 (NF1) and type 2 (NF2), have been distinguished and genetically mapped to different loci, namely chromosomes $17 \mathrm{q} 11.2$ and 22q112 (2). NF1 predominates in neurofibromatosis patients $(\sim 98 \%)$, while NF2 comprises $2 \%$ of this population and is reported as a more rare disease $(1: 35,000)(3)$.

NF1 occurs in $\sim 1$ out of 3,500 births (4) and is defined as an autosomal dominant genetic disorder correlated with point mutations or gross deletions of the NF1 gene (5). Neoplasms in the ends of the limbs are one symptom of NF1. Although uncommon, NF1 patients may exhibit macrodactylia of the fingers or toes accompanied by the proliferation of the vessels, phalanges and subcutaneous fat (6). The exact cellular genes that participate in nerve growth in NF1 patients remain unclear.

In the present study, abnormal bulky terminal nerves were obtained from three diagnosed NF1 patients, and the expression profiles of nerves in the NF1 patients and normal controls were analyzed by microarray analysis. The genes likely to be involved in promoting nerve growth were also identified.

\section{Materials and methods}

Patients and tissues. Three confirmed NF1 patients (male; age, 20-30 years), with macrodactylia of the fingers or toes, and five healthy controls were recruited from the Department of General Surgery at The Third Affiliated Hospital of Southern Medical University (Guangzhou, China) between 2009 and 2011. The healthy controls all underwent amputations due to car accidents or fires. None of the patients and healthy controls 
Table I. Primers for checking relative mRNA levels.

\begin{tabular}{|c|c|c|}
\hline Primer & Sequence & Length, bp \\
\hline \multirow[t]{2}{*}{ ACSBG1 } & Forward: ACGCCATCATTGACACCC & \\
\hline & Reverse: CGTAGCCCGCATACAAGC & 630 \\
\hline \multirow[t]{2}{*}{ ADAMTS4 } & Forward: AGGTCCCATGTGCAACG & \\
\hline & Reverse: TGGAGCCTCTGGTTTGTCT & 724 \\
\hline \multirow[t]{2}{*}{ DGKB } & Forward: GGACCATATTTTACCACC & \\
\hline & Reverse: AACTTCCACCTGTCCAAC & 600 \\
\hline \multirow[t]{2}{*}{ FPR1 } & Forward: TCTCCCCACGAACATCTC & \\
\hline & Reverse: TTGTGGATCTTGGTGGC & 672 \\
\hline \multirow[t]{2}{*}{$\mathrm{HIF} 3 \alpha$} & Forward: CAGTAGCAGCCACTCCCAG & \\
\hline & Reverse: CAGGGGCTCATTCAGGTT & 635 \\
\hline \multirow[t]{2}{*}{$\mathrm{HOXC8}$} & Forward: CTGTTCTCCAAATACAAAGCCG & \\
\hline & Reverse: СТТСТТССТССАССТТСТССТС & 652 \\
\hline \multirow[t]{2}{*}{ IL1 $\beta$} & Forward: GCTTATTACAGTGGCAATGAGG & \\
\hline & Reverse: GGATCTACACTCTCCAGCTGTAGAG & 572 \\
\hline \multirow[t]{2}{*}{ MMP9 } & Forward: CTCCAGAAGCAACTGTCCC & \\
\hline & Reverse: CCATCAGCATTGCCGTC & 635 \\
\hline \multirow[t]{2}{*}{ RCAN1 } & Forward: GTGGCCGGTCCCCAG & \\
\hline & Reverse: TTGGCTTAGGTCTCCTCATTCT & 697 \\
\hline \multirow[t]{2}{*}{ SLC11A1 } & Forward: CCACGACTACGCCAAGAT & \\
\hline & Reverse: AAGGAGCCCATACAGGAAG & 640 \\
\hline
\end{tabular}

were suffering from systemic disorders or viral infections. The nerve tissues of the two groups were separated during surgery. Subsequent to splitting them into small sections, the tissues were powdered in liquid nitrogen and the samples were kept refrigerated at $-80^{\circ} \mathrm{C}$. All study participants provided written informed consent and the study was approved by the Ethics Committee of Jilin University (Changchun, China).

Microarray analysis. RNA was extracted using the RNeasy mini kit (Qiagen, Hilden, Germany). Aliquots of the RNA were analyzed by a Bioanalyser 2100 (Agilent Technologies, Santa Clara, CA, USA) and quantified by a Nano Drop ND-1000 (Labtech International, Uckfield, UK). The Whole Human Genome Microarray (Agilent Technologies) was selected to screen for the expression profiles of the different genes in the NF1 patients and healthy controls. Following hybridization, the microarrays were scanned in the Agilent Microarray Scanner, analyzed with the Feature Extraction software and the raw data were normalized by the Quantile Algorithm. To control the false discovery rate, the Q-value of all genes used in this analysis was assessed using $\mathrm{Q}<0.05$.

Cell culture. PC12 rat pheochromocytoma cells were initially obtained from the American Type Culture Collection (Manassas, VA, USA) and cultured in RPMI-1640 supplemented with $10 \%(\mathrm{v} / \mathrm{v})$ fetal calf serum, cultivated at $37^{\circ} \mathrm{C}$ in a $5 \% \mathrm{CO}_{2}$ incubator. At $70-90 \%$ confluency, the cells $\left(2.5 \times 10^{5}\right)$ were seeded onto 12-well plates (Iwaki, Tokyo, Japan).
Following a 24-h incubation, differentiation was induced by adding $50 \mathrm{ng} / \mathrm{ml}$ nerve growth factor (NGF; Chemicon, Temecula, CA, USA) (7). The medium was changed every two days. Following a 96-h incubation, the PC12 cells were transformed into neuronal-like cells.

RNA extraction and RT-PCR. The PC12 cells were harvested following differentiation and the total RNA was isolated using TRIzol (Invitrogen Life Technologies, Carlsbad, CA, USA) according to the manufacturer's instructions. The RNA pellets were stored in sterile ribonuclease-free water. Reverse transcription was conducted using $1 \mu \mathrm{g}$ total RNA, $0.5 \mu \mathrm{g}$ oligo(dT) and Superscript II enzyme (Gibco, Carlsbad, CA, USA). To check the relative mRNA levels, the primers shown in Table I were used.

Western blotting. The cells were harvested by trypsinization, lysed in RIPA buffer (Aidlab, Beijing, China) on ice for 30 mins and then centrifuged (Eppendorf, Hamburg, Germany) at $4^{\circ} \mathrm{C}$ and $12,000 \mathrm{x}$ g for $10 \mathrm{mins}$. The whole cell lysate was subjected to sodium dodecyl sulfate-polyacrylamide gel electrophoresis and transferred onto polyvinylidene difluoride membranes (Amersham Life Sciences, Arlington Heights, IL, USA). The membranes were incubated with 5\% skimmed dry milk and then with polyclonal goat anti-human primary antibodies [GAPDH, acyl-CoA synthetase bubblegum family member 1 (ACSBG1), hypoxia inducible factor $3 \alpha$ (HIF3 $\alpha$ ) and HOXC8; Santa Cruz Biotechnology, Inc., Santa Cruz, 
Table II. Genes upregulated $>10$-fold and their corresponding functions.

\begin{tabular}{|c|c|c|c|c|}
\hline Probe ID & Gene & Chromosome No. & Fold change in NF1 & Gene function \\
\hline 1660497 & ADAMTS4 & 1 & 0.052 & $\begin{array}{l}\text { Metalloproteases; } \\
\text { inflammation-related }\end{array}$ \\
\hline 1030270 & FPR1 & 19 & 0.0674 & $\begin{array}{l}\text { Inflammation-related; } \\
\text { interacting with calcium ions }\end{array}$ \\
\hline 620239 & RCAN1 & 21 & 0.0685 & Inhibit calcineurin-dependent pathways \\
\hline 840685 & IL1 $\beta$ & 2 & 0.074 & Inflamatory cytokine \\
\hline 5390220 & MMP9 & 16 & 0.0825 & Degradation of the extracellular matrix \\
\hline 780465 & SLC11A1 & 2 & 0.0828 & $\begin{array}{l}\text { Divalent transition metal transporter; } \\
\text { immunological response-related }\end{array}$ \\
\hline 4640059 & $\mathrm{HOXC8}$ & 12 & 10.531 & Modulates the frequency of transcription \\
\hline 4540192 & DGKB & 7 & 10.4188 & $\begin{array}{l}\text { Interacting with calcium ions; } \\
\text { associated with glucose metabolism }\end{array}$ \\
\hline 1110520 & $\mathrm{HIF} 3 \alpha$ & 19 & 12.637 & $\begin{array}{l}\text { Modulate transcription; } \\
\text { responsor of lowered oxygen tension }\end{array}$ \\
\hline 2480730 & ACSBG1 & 15 & 14.536 & Related to metabolic processes of fatty acids \\
\hline
\end{tabular}

ADAMTS4, a disinterin and metalloproteinase with thrombospondin motifs 4; FPR1, formyl peptide receptor 1; RCAN1, regulator of calcineurin 1; IL1 $\beta$, interleukin 1 $\beta$; MMP9, matrix metallopeptidase 9; SLC11A1, solute family carrier 11; DGKB, diacylglycerol kinase; HIF3 $\alpha$, hypoxia inducible factor $3 \alpha$; ACSBG1, acyl-CoA synthetase bubblegum family member 1 .

CA, USA]. Following washing with phosphate-buffered saline with Tween 20 (PBST), the membranes were incubated with horseradish peroxidase-conjugated mouse anti-goat IgG (Santa Cruz Biotechnology Inc.). The immunoreactivity was visualized with the enhanced chemiluminescence system (ECL; Amersham Life Sciences).

Statistical analysis. Statistical analyses were performed using the Stat View software (SAS Institute, Inc., Cary, NC, USA). $\mathrm{P}<0.05$ was considered to indicate a statistically significant difference. Microarray analyses were analyzed with Feature Extraction software 10.7 and the raw data were normalized by the Quantile Algorithm, Gene Spring Software 11.0 (Agilent Technologies). Student's t-tests were used to identify the differentially-expressed genes.

\section{Results}

Differential gene expression profiles in three NF1 patients. To investigate the etiology of nerve growth in the NF1 patients, the genes that shared the same change trends in the NF1 patients were analyzed to try to explain their similar phenotypes, particularly nerve hyperplasia, vessel overgrowth and subcutaneous fat generation. Various genes were compared between each NF1 patient and the healthy controls. From nearly 30,000 genes, 132, 144 and 158 genes were identified to have significant changes $(\mathrm{P}<0.01)$ in the three $\mathrm{NF} 1$ patients, respectively. A common set of 61 genes exhibited the same trend in the three patients, and among these, 28 genes changed markedly $>5$-fold. Gene ontology (GO) analysis revealed that genes associated with DNA/protein binding represented the largest proportion (48\%) of the total number of different genes (Fig. 1), while others involved in regulating molecular transduction $(23 \%)$ and catalysis (18\%) also represented a high percentage.

Confirmation of possible genes in regulating nerve overgrowth of NF1

Transcriptional level. Of the 28 evidently changed genes, the metastasis-associated genes, lymphocyte function-associated antigen 1 (LFA1), C-X-C chemokine receptor type 4 (CXCR4) and intracellular adhesion molecule 1 (ICAM1), and the inflammatory cytokines, interleukin (IL)1 $\beta$, IL6 and IL8, were generally expressed at a low level, and the genes involved in the cell cycle, cyclin-dependent kinase 4 inhibitor, cyclin dependent kinase 4 and Serine/threonine-protein kinase 1, and apoptosis, LMNB1, BIRC3 and FOSL1, were also significantly downregulated. It is noteworthy that $>75 \%$ of these genes were downregulated, and that the majority of them were confirmed to be significant in promoting tumor proliferation and metastasis. Thus, the mechanism of the abnormal overgrowth of the nerves, including the vessels, phalanges and subcutaneous fat around the vessels, in NF1 patients varied from that of cancer tissues. To identify the main factors that affect nerve growth, genes that were up- or downregulated $>10$-fold change were investigated. The screened genes are listed in Table II. The levels of mRNA expression of these genes are shown in Fig. 2.

Protein level. To determine whether the screened genes that changed at the transcriptional level were also altered at the protein level, the expression of ACSBG1, hHIF3 $\alpha$ and HOXC8, which possessed statistical significant changes in mRNA expression was analyzed. By inducing PC12 cells to differentiate into neuron-like cells that were characterized by axon growth, HOXC8 was identified to be upregulated during the process of $\mathrm{PC} 12$ differentiation (Fig. 3). This implies that HOXC8 may be involved in promoting nerve growth in NF1. 


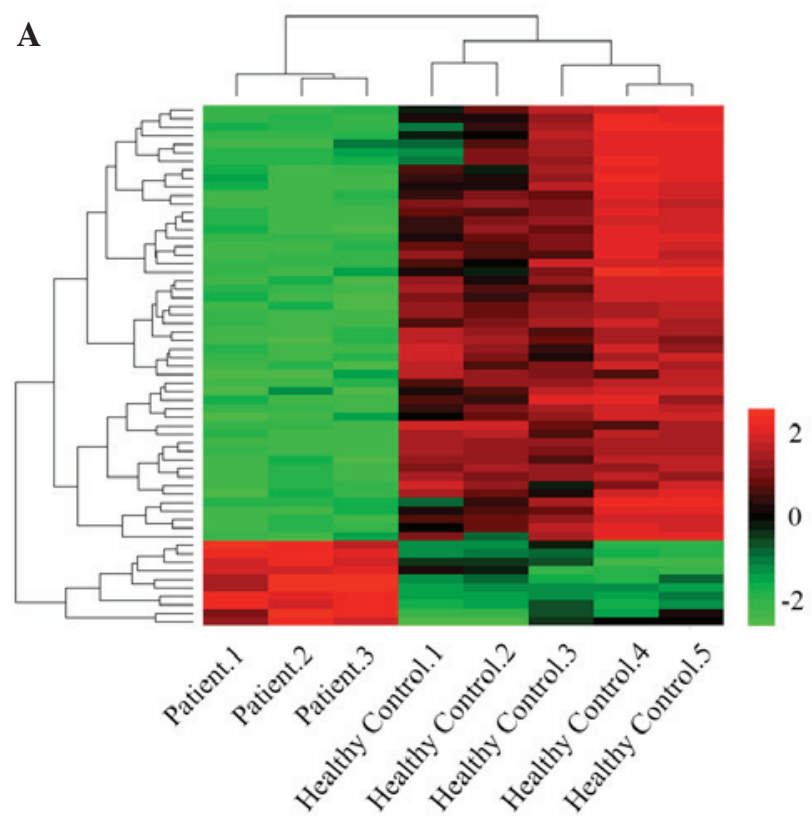

B

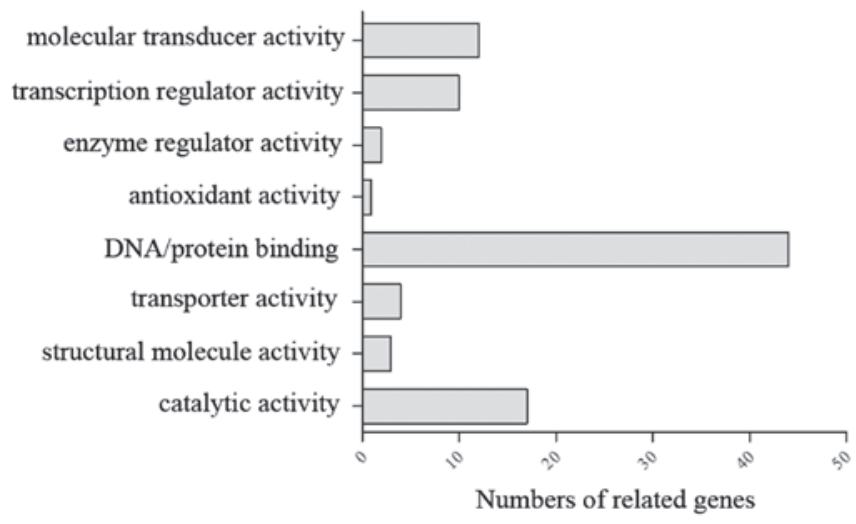

Figure 1. Gene expression profile analysis of patients with NF1. (A) Hierarchical clustering analysis of genes associated with NF1. The red color represents the upregulated genes and the green color represents the downregulated genes. (B) Gene ontology enrichment analysis of differentially expressed genes between the NF1 patients and controls. NF1; neurofibromatosis type 1 .

Location analysis of altered genes. The common locations of genes that shared the same change trend in the three patients were clarified in this study. The results revealed that genes located on chromosomes 1, 2, 12, 17 and 19 possessed the most marked changes in the NF1 patients. The genes on chromosomes 1 and 19 in particular indicated potential gene clusters associated with the nerve growth abnormalities. The chromosomal localization was displayed as shown in Fig. 4.

\section{Discussion}

Although NF1 has attracted attention for a long period of time, the pathogenetic mechanism and intracellular factors involved in nerve growth during NF1 development remain unclear. In the present study, comparisons of gene expression profiles between NF1 and healthy controls were investigated by microarray analysis. A total of 132, 144 and 158 genes from the nerve tissues of the three NF1 patients showed statistically

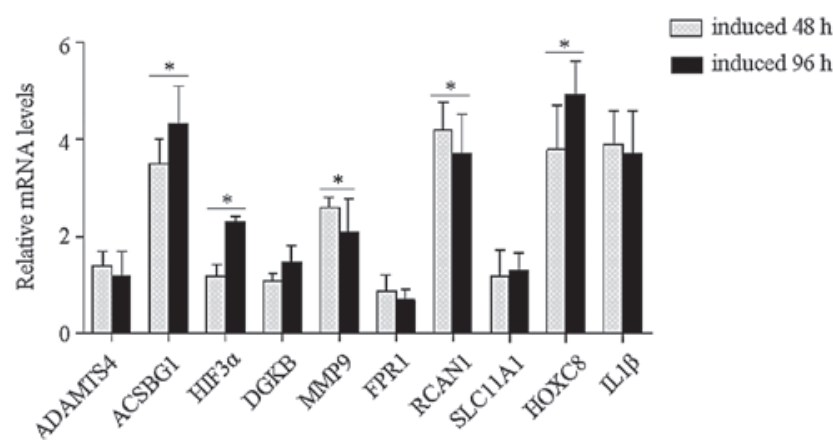

Figure 2. mRNA expression levels of possible genes related with NF1 development. PC12 cells were induced to differentiate using NGF. Following $96 \mathrm{~h}$ of induction, the cells were harvested and the total RNA isolated. The 10 genes that changed $>10$-fold were analyzed, including the calcium ions channel-related factors, FPR1, RCAN1 and DGKB, the inflammatory-related cytokines, IL1 $\beta$ and SLC11A1, the matrix-degrading genes, ADAMTS4 and MMP9, the transcription modulators, $\mathrm{HIF} 3 \alpha$ and $\mathrm{HOXC} 8$, and the metabolism-related factor, ACSBG1. The expression levels were determined with GAPDH as a reference. ACSBG1, HIF3 $\alpha$ and HOXC8 genes showed evident upregulation within the differentiation of the PC12 cells ( $\mathrm{P}<0.05)$. NF1; neurofibromatosis type 1 , NGF; nerve growth factor; ADAMTS4, a disinterin and metalloproteinase with thrombospondin motifs 4; ACSBG1, acyl-CoA synthetase bubblegum family member 1 ; HIF3 $\alpha$, hypoxia inducible factor $3 \alpha$; DGKB, diacylglycerol kinase; MMP9, matrix metallopeptidase 9; FPR1, formyl peptide receptor 1; RCAN1, regulator of calcineurin 1 ; SLC11A1, solute family carrier 11; IL1 $\beta$, interleukin $1 \beta$.

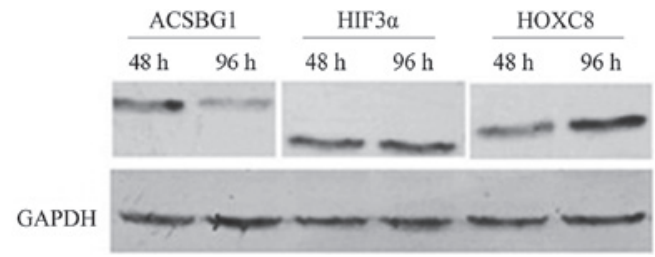

Figure 3. Chromosomal localization of genes that changed $>5$-fold in the NF1 patients. The data showed that the majority of the various genes were located on chromosome 1, 2, 12, 17 and 19, and were particularly focused on chromosome 1 and 19, which affected the development of the nerve system and genetic disease. NF1; neurofibromatosis type 1; ACSBG1, acyl-CoA synthetase bubblegum family member $1 ; \mathrm{HIF} 3 \alpha$, hypoxia inducible factor $3 \alpha$; GAPDH, glyceraldehyde 3-phosphate dehydrogenase.

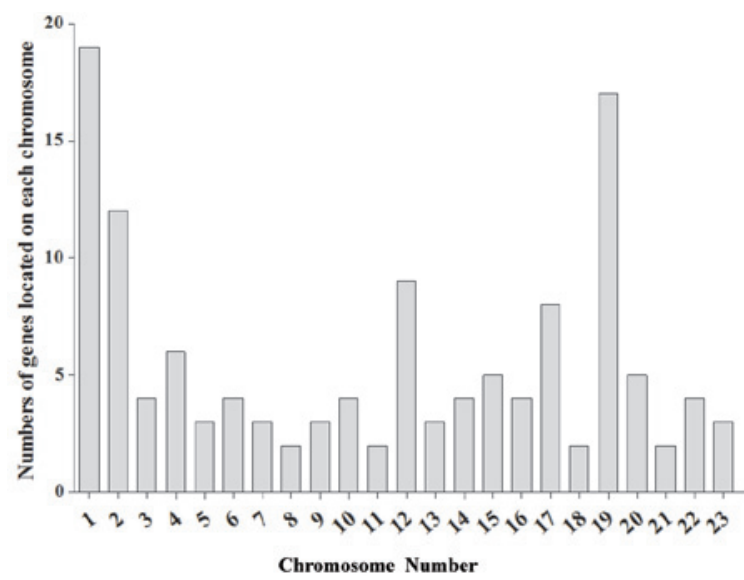

Figure 4. Confirmation of the target protein expression in the PC12 cells. The cells were harvested $96 \mathrm{~h}$ after the PC12 cells were induced by NGF. The protein expression of ACSBG1, HIF3 $\alpha$ and HOXC 8 was determined, and the expression of $\mathrm{HOXC} 8$ was shown to be upregulated following differentiation. This result indicated that HOXC8 may participate in promoting nerve growth in NF1 patients. NF1; neurofibromatosis type 1; ACSBG1, acyl-CoA synthetase bubblegum family member 1 ; HIF $3 \alpha$, hypoxia inducible factor $3 \alpha$. 
significant changes, respectively, and among these genes, downregulated genes accounted for $>70 \%$ in each patient. A total of 28 genes that changed $>5$-fold showed the same trend in all the NF1 patients, including the intracellular protein binding mediators, CXCR4, ICAM1, integrin $\alpha$, LFA1 and major facilitator superfamily domain containing 6 , involved in matrix degradation and metastasis (8), and the inflammatory related factors, IL1 $\beta$, IL6 and IL8, involved in regulating the extracellular microenvironment, angiogenesis and tumorigenesis (9). The general downregulation of the tumor-stimulating factors indicated negative regulation of the immune system and of tumor spreading, which may explain why NF1 has always been considered to be a benign tumor that is less likely to become malignant.

To confirm the most likely genes contributing to the effects on nerve growth in NF1 patients, genes that changed up to 10-fold, including the calcium related factors, formyl peptide receptor 1 (FPR1), regulator of calcineurin 1 (RCAN1) and diacylglycerol kinase, the inflammatory cytokines, IL1 $\beta$ and solute family carrier 11 , the matrix-degrading genes, a disinterin and metalloproteinase with thrombospondin motifs 4 and matrix metallopeptidase, the transcription modulators, HIF3 $\alpha$ and HOXC8, and the metabolism-related factor, ACSBG1, were investigated. With the exception of the inflammatory cytokines and the matrix-degrading genes, which have been reported to be involved in tumor proliferation and metastasis, FPR1 and RCAN1 are expressed in malignant cancers (10-12). The marked downregulation of these genes once again provided evidence that inflammation and basement membrane degradation are suppressed in NF1 patients, which may reduce the risk of tumor metastasis. HIF $3 \alpha$ acts as a negative regulator of hypoxia, so it may induce the tolerance of the extracellular environment (13). Whether HIF3 $\alpha$ is involved in promoting nerve growth remains to be verified. HOXC8 is an essential factor in oncogenesis and tumor metastasis. HOXC8 is downregulated in esophageal and prostate cancers (14), but significantly elevated in metastatic breast cancer cells (15). The complex function of HOXC8 may indicate a temporal role for HOXC8 in tumor progression. To confirm the screened genes that regulate nerve overgrowth, the ACSBG1, HIF3 $\alpha$ and HOXC8 genes that changed markedly at the transcriptional level were checked. The results indicated that HOXC8 exhibited a positive correlation with the differentiation of the $\mathrm{PC} 12$ cells, thus it may be a potential stimulating factor involved in nerve axon growth.

The chromosomal localization of genes exhibiting a $>5$-fold change was also analyzed. This confirmed that genes located on chromosome 1, which affect nerve system development (16), and on chromosome 19, which usually associate with genetic diseases (17), were mainly correlated with the development of NF1. The key cluster of genes that stimulate nerve growth on chromosomes 1 and 19 require identification in future studies.

In conclusion, the etiology of NF1 is complicated and results from changes in multiple genes. Therefore, the identification of such genes is essential in order to explain NF1 formation, metastasis and recurrence. Through large-scale microarray analysis of NF1 patients, possible genes that contribute to the promotion of nerve growth during NF1 development were investigated, which may deepen our understanding of NF1 and provide a novel direction for the research into nerve repair and regeneration.

\section{Acknowledgements}

The authors are grateful to the staff of the Department of General Surgery of The Third Affiliated Hospital of Southern Medical University. This study was supported by a grant from the Key Supporting Project of the Jilin Provincial Science and Technology Department (grant no. 20110456).

\section{References}

1. Lammert M, Friedman JM, Kluwe L and Mautner VF: Prevalence of neurofibromatosis 1 in German children at elementary school enrollment. Arch Dermatol 141: 71-74, 2005.

2. Bausch B, Borozdin W, Mautner VF, et al; European-American Phaeochromocytoma Registry Study Group: Germline NF1 mutational spectra and loss-of-heterozygosity analyses in patients with pheochromocytoma and neurofibromatosis type 1 . J Clin Endocrinol Metab 92: 2784-2792, 2007.

3. Evans DG, Huson SM, Donnai D, et al: A genetic study of type 2 neurofibromatosis in the United Kingdom. I. Prevalence, mutation rate, fitness, and confirmation of maternal transmission effect on severity. J Med Genet 29: 841-846, 1992.

4. Rasmussen SA and Friedman JM: NF1 gene and neurofibromatosis 1. Am J Epidemiol 151: 33-40, 2000.

5. Bottillo I, Ahlquist T, Brekke H, et al: Germline and somatic NF1 mutations in sporadic and NF1-associated malignant peripheral nerve sheath tumours. J Pathol 217: 693-701, 2009.

6. Jouhilahti EM, Peltonen S, Heape AM and Peltonen J: The pathoetiology of neurofibromatosis 1. Am J Pathol 178: 1932-1939, 2011.

7. Nishimura T, Ishima T, Iyo M and Hashimoto K: Potentiation of nerve growth factor-induced neurite outgrowth by fluvoxamine: role of sigma-1 receptors, IP3 receptors and cellular signaling pathways. PLoS One 3: e2558, 2008.

8. Albini A and Sporn MB: The tumour microenvironment as a target for chemoprevention. Nat Rev Cancer 7: 139-147, 2007

9. de Visser KE, Eichten A and Coussens LM: Paradoxical roles of the immune system during cancer development. Nat Rev Cancer 6: 24-37, 2006.

10. RescherU,Danielczyk A, Markoff A and Gerke V: Functional activation of the formyl peptide receptor by a new endogenous ligand in human lung A549 cells. J Immunol 169: 1500-1504, 2002.

11. Kim SS and Seo SR: The regulator of calcineurin 1 (RCAN1/DSCR1) activates the cAMP response element-binding protein (CREB) pathway. J Biol Chem 286: 37841-37848, 2011.

12. Blanco-Mezquita T, Martinez-Garcia C, Proença R, et al: Nerve growth factor promotes corneal epithelial migration by enhancing expression of matrix metalloprotease-9. Invest Ophthalmol Vis Sci. 54: 3880-3890, 2013.

13. Augstein A, Poitz DM, Braun-Dullaeus RC, Strasser RH and Schmeisser A: Cell-specific and hypoxia-dependent regulation of human HIF-3 $\alpha$ : inhibition of the expression of HIF target genes in vascular cells. Cell Mol Life Sci 68: 2627-2642, 2011.

14. Miller GJ, Miller HL, van Bokhoven A, Lambert JR, Werahera PN, Schirripa O, et al: Aberrant HOXC expression accompanies the malignant phenotype in human prostate. Cancer Res 63: 5879-5888, 2003.

15. Li Y, Zhang M, Chen H, Dong Z, Ganapathy V, Thangaraju M and Huang S: Ratio of miR-196s to HOXC8 messenger RNA correlates with breast cancer cell migration and metastasis. Cancer Res 70: 7894-904, 2010.

16. Garlow SJ, Boone E, Kinkead B and Nemeroff CB: Genetic analysis of the hypothalamic neurotensin system. Neuropsychopharmacology 31: 535-543, 2006.

17. Grimwood J, Gordon LA, Olsen A, et al: The DNA sequence and biology of human chromosome 19. Nature 428: 529-535, 2004. 